\title{
Variation in seizure prophylaxis in severe pediatric traumatic brain injury
}

\author{
Paige J. Ostahowski, BA, ${ }^{1}$ Nithya Kannan, MD, ${ }^{2}$ Mark S. Wainwright, MD, PhD, ${ }^{3}$ Qian Qiu, MBA, ${ }^{4}$ \\ Richard B. Mink, MD, MACM, ${ }^{5}$ Jonathan I. Groner, MD, ${ }^{6}$ Michael J. Bell, MD, ${ }^{7}$ \\ Christopher C. Giza, MD, ${ }^{8,9}$ Douglas F. Zatzick, MD, ${ }^{10}$ Richard G. Ellenbogen, MD, ${ }^{11,12}$ \\ Linda Ng Boyle, PhD, ${ }^{13}$ Pamela H. Mitchell, PhD, ${ }^{14}$ and Monica S. Vavilala, MD, ${ }^{11,15}$ \\ for the PEGASUS (Pediatric Guideline Adherence and Outcomes) Study
}

${ }^{1}$ Medical Student Research Training Program, University of Washington School of Medicine; Departments of ${ }^{2}$ Epidemiology, ${ }^{4}$ Pediatrics, ${ }^{10}$ Psychiatry and Behavioral Sciences, ${ }^{11}$ Neurological Surgery, ${ }^{12}$ Global Health Medicine, ${ }^{13}$ Industrial and Systems Engineering, ${ }^{15}$ Anesthesiology \& Pain Medicine, and ${ }^{14}$ School of Nursing, University of Washington, Seattle, Washington; ${ }^{3}$ Department of Pediatrics, Ann \& Robert H. Lurie Children's Hospital of Chicago, Illinois; ${ }^{5}$ Department of Pediatrics, HarborUCLA and Los Angeles BioMedical Research Institute, Torrance; ${ }^{8}$ Department of Neurosurgery and ${ }^{9}$ Division of Pediatric Neurology, Mattel Children's Hospital, UCLA, Los Angeles, California; ${ }^{6}$ Department of Surgery, The Ohio State University College of Medicine, Columbus, Ohio; and 'Department of Critical Care Medicine, University of Pittsburgh, Pennsylvania

\begin{abstract}
OBJECTIVE Posttraumatic seizure is a major complication following traumatic brain injury (TBI). The aim of this study was to determine the variation in seizure prophylaxis in select pediatric trauma centers. The authors hypothesized that there would be wide variation in seizure prophylaxis selection and use, within and between pediatric trauma centers. METHODS In this retrospective multicenter cohort study including 5 regional pediatric trauma centers affiliated with academic medical centers, the authors examined data from 236 children (age < 18 years) with severe TBI (admission Glasgow Coma Scale score $\leq 8$, ICD-9 diagnosis codes of 800.0-801.9, 803.0-804.9, 850.0-854.1, 959.01, 950.1$950.3,995.55$, maximum head Abbreviated Injury Scale score $\geq 3$ ) who received tracheal intubation for $\geq 48$ hours in the ICU between 2007 and 2011.
\end{abstract}

RESULTS Of 236 patients, 187 (79\%) received seizure prophylaxis. In 2 of the 5 centers, $100 \%$ of the patients received seizure prophylaxis medication. Use of seizure prophylaxis was associated with younger patient age $(p<0.001)$, inflicted TBI $(p<0.001)$, subdural hematoma $(p=0.02)$, cerebral infarction $(p<0.001)$, and use of electroencephalography $(p$ $=0.023$ ), but not higher Injury Severity Score. In $63 \%$ cases in which seizure prophylaxis was used, the patients were given the first medication within 24 hours of injury, and $50 \%$ of the patients received the first dose in the prehospital or emergency department setting. Initial seizure prophylaxis was most commonly with fosphenytoin (47\%), followed by phenytoin (40\%).

CONCLUSIONS While fosphenytoin was the most commonly used medication for seizure prophylaxis, there was large variation within and between trauma centers with respect to timing and choice of seizure prophylaxis in severe pediatric TBI. The heterogeneity in seizure prophylaxis use may explain the previously observed lack of relationship between seizure prophylaxis and outcomes.

http://thejns.org/doi/abs/10.3171/2016.4.PEDS1698

KEY WORDS seizures; antiepileptic medications; traumatic brain injury; pediatrics; epilepsy; trauma

$\mathrm{T}$ RAUMATIC brain injury (TBI) is a serious health problem among children in the United States, resulting in over 250,000 emergency department (ED) visits and up to 13,500 hospitalizations annually. ${ }^{23}$ Posttraumatic seizures are a common complication associated with TBI, particularly in cases of severe TBI. ${ }^{17,18,21}$ Since they can cause hypoxia, increased intracranial pressure, increased metabolite production, and higher metabolic demands, seizures may result in secondary brain injury that can have detrimental long-term developmental effects.,12

ABBREVIATIONS AIS = Abbreviated Injury Scale; ED = emergency department; EEG = encephalography; ICU = intensive care unit; ISS = Injury Severity Score; OR = operating room; PEGASUS = Pediatric Guideline Adherence and Outcomes; PICU = pediatric ICU; TBI = traumatic brain injury.

SUBMITTED February 18, 2016. ACCEPTED April 13, 2016.

INCLUDE WHEN CITING Published online June 3, 2016; DOI: 10.3171/2016.4.PEDS1698. 
Therefore, prevention and expedient management of seizures is an important aspect of the care of critically injured children with TBI.

Seizures after TBI can occur early (0-7 days postinjury) or late ( 8 or more days postinjury). ${ }^{13,18,22}$ Early seizure prophylaxis may be associated with a reduced incidence of early posttraumatic seizures which may lead to less secondary neurological insult and better postdischarge outcomes. Observational studies suggest that decreased posttraumatic seizures may have a survival benefit in patients treated with phenytoin in the acute phase. . $0,17,18,22,24^{2}$ These findings served as the rationale for the 2012 Brain Trauma Foundation Guidelines, which detail acute care recommendations for children with severe TBI. While the Guidelines recommend phenytoin as the seizure prophylaxis medication of choice to prevent early seizures, ${ }^{1,16}$ there were only a small number of studies that were eligible to serve as evidence (3 Level III studies for the 2003 Guidelines, and only 1 Level III study for the 2012 updated Guidelines) for this recommendation. Moreover, recommendations pertaining to seizure prophylaxis such as time of medication initiation and choice of medication were not specifically considered, leaving these decisions to provider discretion.

The specific mechanisms by which seizure prophylaxis may or may not affect cerebral outcomes is not yet known. The recent Pediatric Guideline Adherence and Outcomes (PEGASUS) study, which examined the relationship between adherence to the 2003 Brain Trauma Foundation Guidelines and patient outcomes, reported a relationship between guideline adherence and favorable patient outcomes. ${ }^{25}$ In this study, there was no relationship between seizure prophylaxis examined in the intensive care unit and outcomes. However, this study was not powered to examine the effects of seizure prophylaxis use on outcomes and hence may have resulted in the null findings. Therefore, we aimed to examine the characteristics of seizure prophylaxis use in the PEGASUS cohort. Given the lack of strong evidence in the Guidelines and our observed lack of association between seizure prophylaxis use and outcomes, we hypothesized that there would be a wide variation in the use of seizure prophylaxis at various locations as well as the choice of medication used for seizure prophylaxis both within and between leading pediatric trauma centers.

\section{Methods}

This study was approved by the University of Washington institutional review board.

\section{Data Set and Study Population}

This study is a focused analysis of a data set collected from 2007 to 2012 for the PEGASUS study. ${ }^{25}$ Data were collected retrospectively and includes 236 records from 5 urban pediatric trauma centers affiliated with academic medical centers located in 5 different U.S. states. The pediatric trauma centers that contributed patient data are the University of Washington's Harborview Medical Center, Seattle, WA; Children's Hospital of Pittsburgh, Pittsburgh, PA; Ann and Robert H. Lurie Children's Hospital of Chi- cago, Chicago, IL; Harbor University of California, Los Angeles Medical Center, Torrance, CA; and Nationwide Children's Hospital, Columbus, $\mathrm{OH}$. Each center was able to contribute data on 30 to 50 pediatric patients with severe TBI. All patients were under 18 years of age and presented with severe TBI as defined by an ICD-9-CM code indicating TBI, Glasgow Coma Scale Score of less than 9, a minimum head Abbreviated Injury Scale (AIS) score $\geq 3$, and history of trauma. Eligible patients were also required to be in the intensive care unit (ICU) and intubated for at least 48 hours and have an abnormal head CT upon admission (subdural hematoma, intracranial hemorrhage, intraventricular hemorrhage, cerebral infarction, diffuse axonal injury). ${ }^{25}$

\section{Seizure Prophylaxis Post-TBI}

The following seizure prophylaxis data were abstracted: patient demographics, injury and treatment characteristics, seizure prophylaxis used (single and multiple [serial administration] medications used), time from trauma center admission to first use of seizure prophylaxis, overall seizure prophylaxis use among the participating centers, and seizure prophylaxis of choice by trauma center and treatment location (prehospital, ED, operating room [OR], and pediatric ICU [PICU]). Since the hour of initiation of seizure prophylaxis was not recorded, time to first seizure prophylaxis medication use was used as an approximation based on the date of injury and first seizure prophylaxis used. When examining types of seizure prophylaxis used, only those patients actually receiving at least 1 dose of medication for seizure prophylaxis were included in the analysis. For the purpose of this study, "prophylactic" refers to seizure prophylaxis that was administered with the intent to prevent seizures.

\section{Statistical Analysis}

Descriptive statistics were used to characterize clinical characteristics of the cohort. Frequencies and percentages were calculated for the data collected and were compared within each center and between centers. Patient and injury characteristics were examined in bivariate analyses with the cases stratified by seizure prophylaxis and by number of seizure medications using chi-square tests for categorical variables and t-tests for continuous variables. Statistical significance was defined as $\mathrm{p}<0.05$. Univariate regression analysis was performed, with adjustments made to correct for the clustering effect between study centers.

\section{Results}

\section{Clinical Characteristics and Seizure Prophylaxis}

The clinical characteristics of the entire cohort of 236 patients have been previously described. ${ }^{25}$ In brief, the average age was 8.0 years and the majority of the patients were male (69\%). One hundred and seventy (72\%) of 236 patients had extracranial injuries. All patient records from each of the 5 centers in the PEGASUS dataset had information pertaining to seizure prophylaxis. Of the 236 patients treated at the 5 participating centers, 187 (79\%) received at least 1 seizure prophylaxis medication, while 
$21 \%$ did not receive any seizure prophylaxis. A comparison of the characteristics of patients who did and did not receive seizure prophylaxis medication is provided in Table 1 . In 2 of the 5 centers, $100 \%$ of the patients with severe TBI received seizure prophylaxis medication.

Seizure prophylaxis was associated with younger age (mean age 7.0 [SD 6.0]), inflicted injury (30\%), presence of subdural hematoma or cerebral infarction on CT scan (70\% and 19\%, respectively), and use of electroencephalography (EEG, 66\%). Overall, neither greater Injury Severity Score (ISS) nor head AIS score was associated with seizure prophylaxis use. The majority of patients who did not receive seizure prophylaxis had an admission motor Glasgow Coma Scale (GCS) of 1 ( $p<0.001)$.

\section{Time and Location of Seizure Prophylaxis}

Time from injury to first seizure prophylaxis use is giv- en in Fig. 1. Over half (63\%) of the patients receiving any seizure prophylaxis were given an initial dose within 24 hours. In almost $95 \%$ of patients, seizure prophylaxis was initiated within 72 hours of injury.

The location of seizure prophylaxis initiation varied by center and within each center. Of the 187 patients who received at least 1 prophylactic dose of antiseizure medication, approximately $44 \%$ received the first dose in the PICU. Seizure prophylaxis was initiated in the OR in 11 patients in $4(60 \%)$ of 5 centers. Collectively, about half of the patients treated with seizure prophylaxis received their first dose of antiseizure medication in the prehospital or ED setting (Fig. 2).

\section{Choice of Seizure Prophylaxis by Center and Location}

The choice of initial seizure prophylaxis varied with-

TABLE 1. Clinical characteristics associated with any seizure prophylaxis medication use*

\begin{tabular}{|c|c|c|c|c|}
\hline \multirow[b]{2}{*}{ Variable } & \multirow[b]{2}{*}{ Total } & \multicolumn{2}{|c|}{ Seizure Prophylaxis Medication Used } & \multirow[b]{2}{*}{$p$ Value $†$} \\
\hline & & Yes & No & \\
\hline No. of patients & 236 & $187(79.2)$ & $49(20.8)$ & \\
\hline Mean age in yrs [SD] & $8.0[6.3]$ & $7.0[6.0]$ & $12.1[5.4]$ & $<0.001$ \\
\hline Male sex & $163(69.1)$ & $128(68.5)$ & $35(71.4)$ & 0.532 \\
\hline Mean GCS score at admission [SD] & $4.61[1.82]$ & $4.95[1.84]$ & $3.35[1.01]$ & $<0.001$ \\
\hline Motor GCS score at admission & & & & $<0.001$ \\
\hline 1 & $118(50)$ & $75(40.1)$ & $43(87.8)$ & \\
\hline 2 & $11(4.7)$ & $11(5.9)$ & $0(0.0)$ & \\
\hline 3 & $21(8.9)$ & $18(9.6)$ & $3(6.3)$ & \\
\hline 4 & $43(18.2)$ & $41(21.8)$ & $2(4.2)$ & \\
\hline 5 & $39(16.5)$ & $39(20.7)$ & $0(0.0)$ & \\
\hline 6 & $4(1.7)$ & $3(1.6)$ & $1(2.1)$ & \\
\hline Mechanism of injury & & & & $<0.001$ \\
\hline Nonaccidental trauma & $58(24.6)$ & $56(30.0)$ & $2(4.1)$ & \\
\hline Accidental trauma & $178(75.4)$ & $131(70.0)$ & $47(95.9)$ & \\
\hline Head AIS score & & & & 0.052 \\
\hline 3 & $28(11.9)$ & $23(12.3)$ & $5(10.2)$ & \\
\hline 4 & $84(35.6)$ & $73(39.0)$ & $11(22.5)$ & \\
\hline 5 & $121(51.3)$ & $89(47.6)$ & $32(65.3)$ & \\
\hline 6 & $3(1.3)$ & $2(1.1)$ & $1(2.0)$ & \\
\hline Mean ISS [SD] & $28.3[12.8]$ & $25.7[11.2]$ & $38.2[13.8]$ & 0.01 \\
\hline \multicolumn{5}{|l|}{ Head CT } \\
\hline Epidural hematoma & $40(17.0)$ & $31(16.6)$ & $9(18.4)$ & 0.74 \\
\hline Subdural hematoma & $157(66.5)$ & $131(70.1)$ & $26(53.1)$ & 0.02 \\
\hline Subarachnoid hemorrhage & $108(45.8)$ & $82(43.9)$ & $26(53.1)$ & 0.574 \\
\hline Intracerebral hemorrhage & $74(31.4)$ & $52(27.8)$ & $22(44.9)$ & 0.001 \\
\hline Intraventricular hemorrhage & $47(19.9)$ & $31(16.6)$ & $16(32.7)$ & $<0.001$ \\
\hline Cerebral edema & $127(53.8)$ & $101(54.0)$ & $26(53.1)$ & 0.925 \\
\hline Cerebral infarction & $39(16.5)$ & $36(19.3)$ & $3(6.1)$ & $<0.001$ \\
\hline Contusion & $94(39.8)$ & $78(41.7)$ & $16(32.7)$ & 0.336 \\
\hline Diffuse axonal injury & $70(29.7)$ & $48(25.7)$ & $22(44.9)$ & 0.029 \\
\hline Surgery performed & $151(64.0)$ & 118 (63.1) & $33(67.4)$ & 0.499 \\
\hline EEG performed & $136(57.6)$ & $123(65.8)$ & $13(26.5)$ & 0.023 \\
\hline
\end{tabular}

* Values are numbers of patients (\%) unless otherwise indicated.

$\dagger$ Univariate regression analysis adjusted for clustering effect of centers. Boldface type indicates statistical significance. 


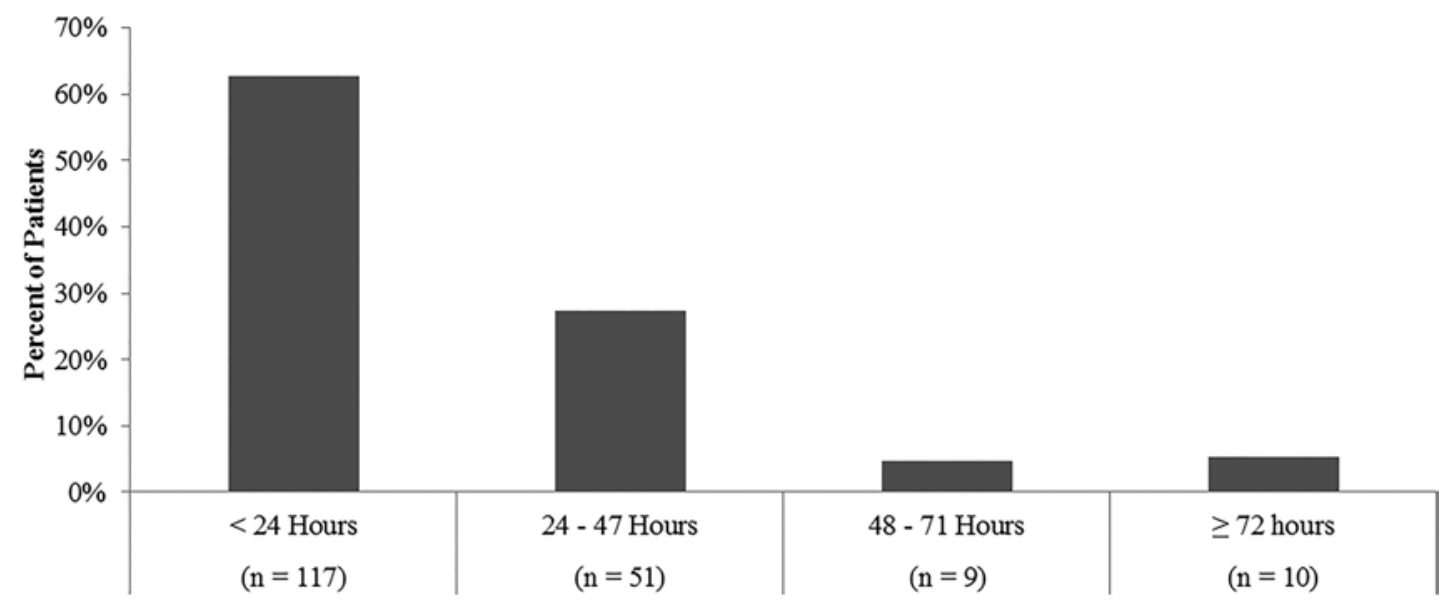

FIG. 1. Bar graph showing the time to initiation of seizure prophylaxis medication after TBI in 187 children across 5 centers.

in and between centers and treatment locations (Fig. 3, Supplementary Fig. 1). Across all centers, 4 different antiseizure medications were used for initiation of seizure prophylaxis. Fosphenytoin was the most commonly used (47\%), and phenytoin was the second most used (40\%). There was within-center variation in initial seizure prophylaxis choice, with 4 of 5 centers using at least 3 different antiseizure medications for initial prophylaxis. Center 5 differed from the other 4 centers with regard to location of initiation of seizure prophylaxis and choice of initial seizure prophylaxis, with over $95 \%$ of the patients receiving phenytoin as the first drug of choice and over $80 \%$ receiving their first dose of antiseizure medication in the ED. The most variation with regard to choice of prophylactic seizure medication use within centers occurred at Centers 1, 3, and 4, where all 4 different antiseizure medications were used. There was also variation in drug choice across treatment locations (Supplementary Fig. 1). Phenytoin, fosphenytoin, and phenobarbital were used in all locations, while levetiracetam was limited to the PICU. Phenytoin was favored as the initial seizure prophylaxis medication in the ED and OR (used in $68 \%$ and $82 \%$ of cases, respectively) but was only used in $12 \%$ of cases in the PICU, where fosphenytoin was the favored (used in $64 \%$ of cases).

\section{Use of Single Versus Multiple Seizure Prophylaxis Medications}

The clinical characteristics of patients receiving single versus multiple types of seizure prophylaxis were similar (Table 2). Twenty-three (12\%) of 187 patients receiving any seizure prophylaxis were given multiple types during their treatment. Subdural hematoma and intraventricular hemorrhage were each associated with use of multiple

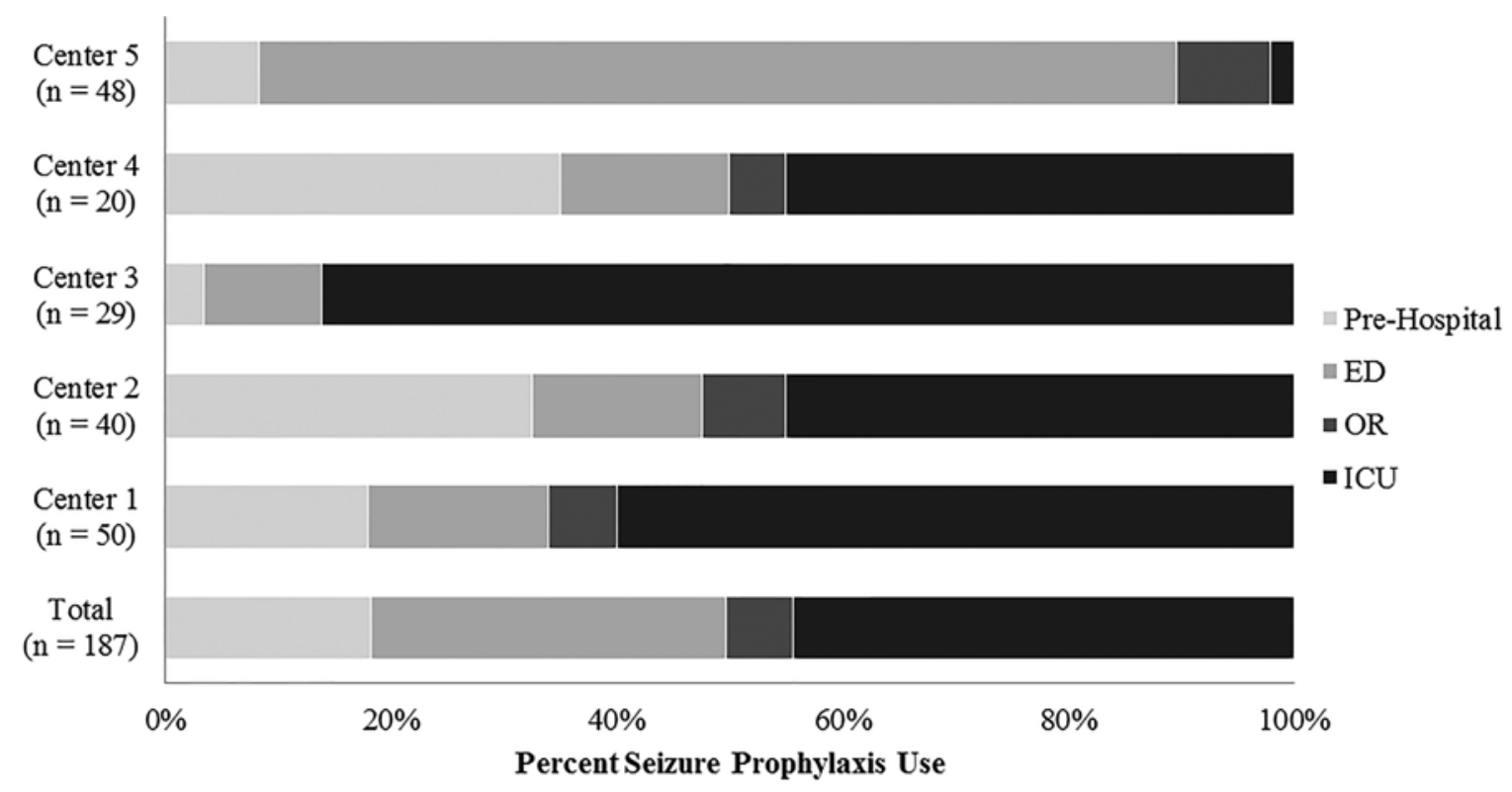

FIG. 2. Bar graph showing the location of initiation of seizure prophylaxis medication after TBI in 187 children by center. 


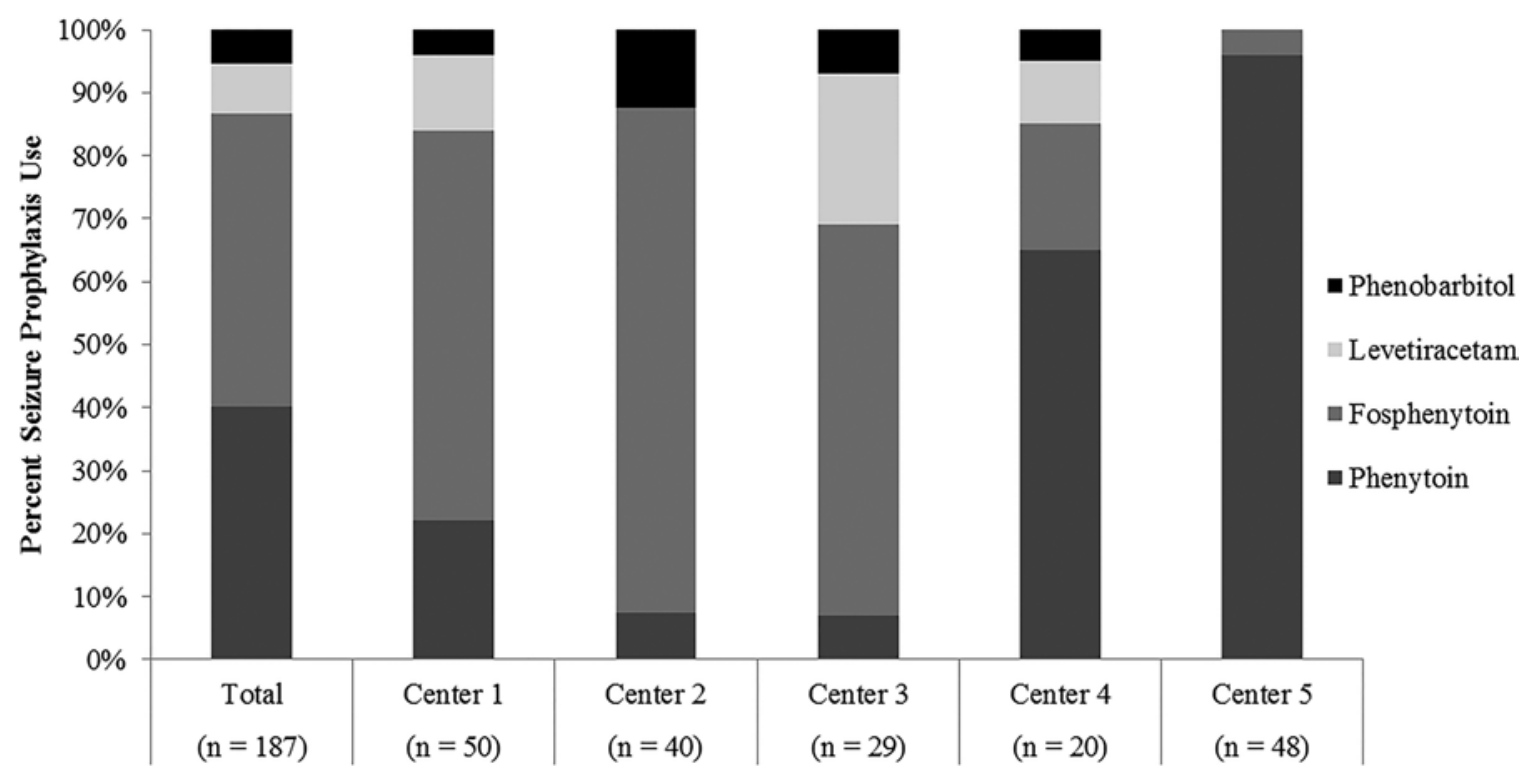

FIG. 3. Stacked bar graph showing the choice of initial seizure prophylaxis medication after TBI by center in 187 children.

prophylactic antiseizure medications. Use of any seizure prophylaxis medication, age, mechanism of injury, and overall injury severity were not significantly associated with the use of multiple seizure prophylaxis types.

Between-centers analysis of seizure prophylaxis use and associated factors are shown in Table 3. In all centers except Center 4, most patients (70\%-92\%) were treated with a single antiseizure medication for seizure prophylaxis. There was a statistically significant difference in use and choice of prophylactic antiseizure medications by center $(\mathrm{p}<0.001)$, both overall and when compared with associated factors (age, inflicted TBI, cerebral infarction, any encephalography [EEG]).

Electroencephalography was performed in 136 (58\%) of 236 cases. Of the 187 patients who received seizure prophylaxis, 123 (66\%) underwent EEG. Of the 49 patients who did not receive seizure prophylaxis medication, 13 (28\%) underwent EEG; 9 (69\%) of these 13 patients were from Center 4. There was a wide variation in EEG utilization among those patients who received seizure prophylaxis (14\%-100\% of patients across the 5 centers). Centers 1 and 2 had higher EEG utilization rates with increased use of seizure prophylaxis, while Centers 3, 4, and 5 had lower EEG utilization.

\section{Change in Seizure Prophylaxis Use}

There was wide variation in choice of seizure prophylaxis for the 23 patients who received multiple types of seizure prophylaxis. Change from phenytoin to fosphenytoin was the most common (48\%). A total of 7 drug combinations including multiple drug types were used for seizure prophylaxis across the 5 centers.

\section{Discussion}

The Brain Trauma Foundation Guidelines for children with severe TBI recommend the use of phenytoin for early seizure prevention. ${ }^{16}$ However, this recommendation is largely based on a single Level III study published in 1993 that reported a decrease in early seizure rates among those children treated with phenytoin. ${ }^{17}$ As a result, the Guidelines do not address the specifics of seizure prophylaxis, such as timing, choice, or which patients should be considered the appropriate recipients of seizure prophylaxis. The main findings of this study are as follows: 1) Although fosphenytoin was the most commonly used medication for initiation of seizure prophylaxis, there was large variation in seizure prophylaxis use within and between centers and across treatment locations. 2) The majority of patients received initial seizure prophylaxis early after severe TBI. 3) The use of multiple types of seizure prophylaxis medications was associated with intraventricular hemorrhage and diagnosis of subdural hematoma.

Some of our results on seizure prophylaxis support previous work showing that younger patients and those with inflicted TBI have higher rates of seizure. ${ }^{4,6,18,20,21}$ Surprisingly, in this study, those patients with a lower ISS were more likely to receive seizure prophylaxis, and this is in contrast to previous studies that have found patients with more severe injury to be at risk for seizure, indicating that measures for seizure prophylaxis would have been used in this group. ${ }^{2,5,18,21}$ In our study, fosphenytoin was the most common initial seizure prophylaxis, which is contradictory to the current recommendations. While phenytoin is the recommended medication according to the Guidelines, studies evaluating the efficacy and cost-effectiveness of the 2 medications do not unanimously support equivalent use. Phenytoin has been linked with a higher incidence of postinfusion sequelae, such as rash and Stevens-Johnson syndrome. ${ }^{11,15}$ However, fosphenytoin is up to 18 times more expensive per dose than phenytoin. Levetiracetam has only been researched as a potential option for seizure prophylaxis, $, 7,13,14,19$ and initial studies have found it as effective as phenytoin but without similar side effects or the need to check drug levels in the blood. ${ }^{7,8,13,14,19,22}$ Even with 
TABLE 2. Clinical characteristics associated with single versus multiple seizure prophylaxis medication*

\begin{tabular}{|c|c|c|c|c|}
\hline Variable & Total & Single Medication & Multiple Medications & $p$ Value $\dagger$ \\
\hline No. of patients & 187 & $164(87.7)$ & $23(12.3)$ & \\
\hline Mean age in yrs [SD] & $7.0[6.0]$ & $7.0[6.0]$ & $7.0[6.3]$ & 0.977 \\
\hline Male sex & $128(68.5)$ & $114(69.5)$ & $14(60.9)$ & 0.514 \\
\hline Mean GCS score at admission [SD] & $4.9[1.8]$ & $5.0[1.8]$ & $4.5[1.7]$ & 0.285 \\
\hline Motor GCS score at admission & & & & 0.2775 \\
\hline 1 & $75(40.1)$ & $64(39.0)$ & $11(47.8)$ & \\
\hline 2 & $11(5.9)$ & $8(4.9)$ & $3(13.0)$ & \\
\hline 3 & $18(9.6)$ & $17(10.4)$ & $1(4.4)$ & \\
\hline 4 & $41(21.9)$ & $38(23.2)$ & $3(13.0)$ & \\
\hline 5 & $39(20.9)$ & $34(20.7)$ & $5(21.7)$ & \\
\hline 6 & $3(1.6)$ & $3(1.8)$ & $0(0.0)$ & \\
\hline Mechanism of injury & & & & 0.332 \\
\hline Nonaccidental trauma & $56(30.0)$ & $48(29.3)$ & $8(34.8)$ & \\
\hline Accidental trauma & $131(70.0)$ & $116(70.7)$ & $15(65.2)$ & \\
\hline \multicolumn{5}{|l|}{ Head AIS score } \\
\hline 3 & $23(12.3)$ & $22(13.4)$ & $1(4.4)$ & 0.2049 \\
\hline 4 & $73(39.0)$ & $63(38.4)$ & $10(43.5)$ & \\
\hline 5 & $89(47.6)$ & $78(47.6)$ & $11(47.8)$ & \\
\hline 6 & $2(1.1)$ & $1(0.6)$ & $1(4.4)$ & \\
\hline Mean ISS [SD] & $25.6[11.2]$ & $25.4[10.8]$ & $27.2[13.4]$ & 0.159 \\
\hline \multicolumn{5}{|l|}{ Head CT } \\
\hline Epidural hematoma & $31(16.6)$ & $29(17.7)$ & $2(8.7)$ & 0.242 \\
\hline Subdural hematoma & $131(70.1)$ & $111(67.7)$ & $20(87.0)$ & 0.008 \\
\hline Subarachnoid hemorrhage & $82(43.9)$ & $73(44.5)$ & $9(39.1)$ & 0.64 \\
\hline Intracerebral hemorrhage & $52(27.8)$ & $46(28.1)$ & $6(26.1)$ & 0.807 \\
\hline Intraventricular hemorrhage & $31(16.6)$ & $24(14.6)$ & $7(30.4)$ & 0.007 \\
\hline Cerebral edema & $101(54.0)$ & $86(52.4)$ & $15(65.2)$ & 0.181 \\
\hline Cerebral infarction & $36(19.3)$ & $29(17.7)$ & $7(30.4)$ & 0.184 \\
\hline Contusion & $78(41.7)$ & $68(41.5)$ & $10(43.5)$ & 0.779 \\
\hline Diffuse axonal injury & $48(25.7)$ & $45(27.4)$ & $3(13.0)$ & 0.337 \\
\hline Surgery performed & $118(63.1)$ & $99(60.4)$ & $19(82.6)$ & 0.17 \\
\hline EEG performed & $123(65.8)$ & $105(64.0)$ & $18(78.3)$ & 0.075 \\
\hline \multicolumn{5}{|l|}{ GOS score at discharge } \\
\hline 1 (death) & $26(13.9)$ & $21(12.8)$ & $5(21.7)$ & 0.169 \\
\hline 2 (vegetative state) & $13(7.0)$ & $12(7.3)$ & $1(4.4)$ & \\
\hline 3 (moderate disability) & $84(44.9)$ & $75(45.7)$ & $9(39.1)$ & \\
\hline 4 (mild disability) & $49(26.2)$ & $43(26.2)$ & $6(26.1)$ & \\
\hline 5 (baseline function) & $15(8.0)$ & $13(7.9)$ & $2(8.7)$ & \\
\hline
\end{tabular}

GOS = Glasgow Outcome Scale.

* Values are numbers of patients (\%) unless otherwise indicated.

$\dagger$ Boldface type indicates statistical significance.

this benefit, levetiracetam may not be used as commonly due to its higher cost relative to phenytoin. ${ }^{13}$

Most patients received seizure prophylaxis within 24 hours of hospital admission and over half received the first dose of medication in the prehospital or ED setting, early in severe TBI care. This is important because studies have reported that first posttraumatic seizures are common within 24 hours of injury. ${ }^{5,15}$ However, although most patients received seizure prophylaxis early, the prehospital setting had a large variation in type of seizure prophylaxis used. The Pediatric Guidelines do not address seizure prophylaxis in the prehospital or OR setting despite the fact that seizure prophylaxis choice may be particularly important early after severe TBI. Interestingly, our study indicates lack of uniformity in seizure prophylaxis choice within and between centers, and also for any given patient at a given pediatric trauma center, demonstrating the lack of consensus as to seizure prophylaxis choice. This pat- 
TABLE 3. Variation in seizure prophylaxis medication use and significantly associated factors by center*

\begin{tabular}{|c|c|c|c|c|c|c|c|}
\hline Group \& Variable & Total & Center 1 & Center 2 & Center 3 & Center 4 & Center 5 & $p$ Value $\dagger$ \\
\hline All patients & 236 & $50(21.2)$ & $42(17.8)$ & $40(17.0)$ & $56(23.7)$ & $48(20.3)$ & $<0.001$ \\
\hline No seizure prophylaxis medication & $49(20.8)$ & $0(0.0)$ & $2(4.8)$ & $11(27.5)$ & $36(64.3)$ & $0(0.0)$ & \\
\hline Single medication type & $164(69.5)$ & $40(80.0)$ & $35(83.3)$ & $28(70.0)$ & $17(30.4)$ & $44(91.7)$ & \\
\hline Multiple medication types & $23(9.8)$ & $10(20.0)$ & $5(11.9)$ & $1(2.5)$ & $3(5.4)$ & $4(8.3)$ & \\
\hline $\begin{array}{l}\text { Patients receiving any seizure prophylaxis } \\
\text { medication }\end{array}$ & $187(79.2)$ & $50(100.0)$ & $40(95.2)$ & $29(72.5)$ & $20(35.7)$ & $48(100.0)$ & \\
\hline Mean age in yrs [SD] & $7.0[6.0]$ & $8.5[5.6]$ & $3.2[4.7]$ & $4.3[5.3]$ & $9.5[6.2]$ & $9.0[5.9]$ & $<0.001$ \\
\hline Nonaccidental trauma & $56(30.0)$ & $13(26.0)$ & $21(52.5)$ & $13(44.8)$ & $1(5.0)$ & $8(16.7)$ & $<0.001$ \\
\hline Subdural hematoma & $131(70.1)$ & $36(72.0)$ & $29(72.5)$ & $24(82.8)$ & $15(75.0)$ & $27(56.3)$ & 0.134 \\
\hline Cerebral infarction & $36(19.3)$ & $13(26.0)$ & $10(25.0)$ & $2(6.9)$ & $7(35.0)$ & $4(8.3)$ & 0.017 \\
\hline EEG performed & $123(65.8)$ & $50(100.0)$ & $38(95.0)$ & $14(48.3)$ & $8(40.0)$ & $13(27.1)$ & $<0.001$ \\
\hline $\begin{array}{l}\text { Patients receiving multiple seizure prophy- } \\
\text { laxis medications }\end{array}$ & $23(9.8)$ & $10(20.0)$ & $5(11.9)$ & $1(2.5)$ & $3(5.4)$ & $4(8.3)$ & \\
\hline Subdural hematoma & $20(87.0)$ & $9(90.0)$ & $5(100.0)$ & $1(100.0)$ & $2(66.7)$ & $3(75.0)$ & 0.631 \\
\hline EEG performed & $18(78.3)$ & $10(100.0)$ & $5(100.0)$ & $0(0.0)$ & $1(33.3)$ & $2(50.0)$ & 0.01 \\
\hline Nonaccidental trauma & $8(34.8)$ & $4(40.0)$ & $3(60.0)$ & $1(100.0)$ & $0(0.0)$ & $0(0.0)$ & 0.129 \\
\hline
\end{tabular}

* Values are numbers of patients (\%) unless otherwise indicated.

$\dagger$ Boldface type indicates statistical significance.

tern reflects not only variation in institutional practice but also variation in practice between clinicians of different disciplines. The observed change in seizure prophylaxis may be problematic if therapeutic levels are not able to be achieved due to this change.

Electroencephalographic monitoring is becoming increasingly common for patients with TBI due to the recognition of the occurrence of subclinical seizures. ${ }^{3,4,9,20}$ In one study, subclinical seizures, confirmed by EEG, occurred in $16.1 \%$ of patients admitted to a PICU with TBI, with $7.5 \%$ of the patient population experiencing only subclinical seizures. ${ }^{4}$ Our study suggests that there was a large variation in EEG utilization across centers, which may suggest differences in institutional practice, provider discretion, and lack of uniform guidelines. It is likely that patients in whom EEG was performed were more likely to receive seizure prophylaxis, although we do not know which occurred first as we do not have additional data on timing of EEG. More patients received seizure prophylaxis than underwent EEG in our cohort, which suggests that seizure prophylaxis was started based on multiple factors, such as younger age, nonaccidental trauma, and/or CT findings, or started empirically depending on provider discretion, which may explain the wide intercenter and intracenter variability in seizure prophylaxis.

There are some limitations to this study. First, data were collected specifically for the PEGASUS study to assess pediatric guideline adherence rather than the specifics of seizure prophylaxis. Second, we did not have information pertaining to actual seizure rates or EEG data, seizure prophylaxis complications, or side-effects of prophylactic seizure medication use in this population. Third, while information is available pertaining to the specific seizure prophylaxis that was initiated in a particular location, the duration of use of each drug was not recorded. Therefore, we focused on the initial seizure prophylaxis administered rather than the total number of doses throughout treatment. Finally, although combining data for phenytoin and fosphenytoin would have reduced the variability in our data set, we did not combine the data for these two drugs because they are 2 distinct medications with different patterns of clinical use.

\section{Conclusions}

In conclusion, this study describes the wide variation in the use of seizure prophylaxis medications in children with severe TBI. Although the current Pediatric Guidelines recommend only phenytoin for use in early seizure prevention, we found large variation in choice of seizure prophylaxis not only between centers but also across treatment locations within centers. The lack of understanding of specific mechanisms by which early seizure prophylaxis may benefit outcomes may explain the heterogeneity in use of seizure prophylaxis medications and the previously observed lack of relationship in the PEGASUS study. Study of the most efficacious seizure prophylaxis medication is warranted in pediatric TBI.

\section{Acknowledgments}

This study was funded by a grant from the National Institute of Neurological Disorders and Stroke (R01 NS072308-05 to M.S.V.).

\section{References}

1. Adelson PD, Bratton SL, Carney NA, Chesnut RM, du Coudray HE, Goldstein B, et al: Guidelines for the acute medical management of severe traumatic brain injury in infants, children, and adolescents. Chapter 19. The role of anti-seizure prophylaxis following severe pediatric traumatic brain injury. Pediatr Crit Care Med 4 (3 Suppl):S72-S75, 2003

2. Annegers JF, Hauser WA, Coan SP, Rocca WA: A population-based study of seizures after traumatic brain injuries. $\mathbf{N}$ Engl J Med 338:20-24, 1998 
3. Arango JI, Deibert CP, Brown D, Bell M, Dvorchik I, Adelson PD: Posttraumatic seizures in children with severe traumatic brain injury. Childs Nerv Syst 28:1925-1929, 2012

4. Arndt DH, Lerner JT, Matsumoto JH, Madikians A, Yudovin $\mathrm{S}$, Valino $\mathrm{H}$, et al: Subclinical early posttraumatic seizures detected by continuous EEG monitoring in a consecutive pediatric cohort. Epilepsia 54:1780-1788, 2013

5. Ateş O, Ondül S, Onal C, Büyükkiraz M, Somay H, Çayli $\mathrm{SR}$, et al: Post-traumatic early epilepsy in pediatric age group with emphasis on influential factors. Childs Nerv Syst 22:279-284, 2006

6. Fulkerson DH, White IK, Rees JM, Baumanis MM, Smith JL, Ackerman LL, et al: Analysis of long-term (median 10.5 years) outcomes in children presenting with traumatic brain injury and an initial Glasgow Coma Scale score of 3 or 4 . $\mathbf{J}$ Neurosurg Pediatr 16:410-419, 2015

7. Gabriel WM, Rowe AS: Long-term comparison of GOS-E scores in patients treated with phenytoin or levetiracetam for posttraumatic seizure prophylaxis after traumatic brain injury. Ann Pharmacother 48:1440-1444, 2014

8. Goraya JS, Khurana DS, Valencia I, Melvin JJ, Cruz M, Legido A, et al: Intravenous levetiracetam in children with epilepsy. Pediatr Neurol 38:177-180, 2008

9. Greiner MV, Greiner HM, Caré MM, Owens D, Shapiro R, Holland K: Adding insult to injury: nonconvulsive seizures in abusive head trauma. J Child Neurol 30:1778-1784, 2015

10. Guilliams K, Wainwright M: Pathophysiology and management of moderate and severe traumatic brain injury in children. J Child Neurol 31:35-45, 2016

11. Haltiner AM, Newell DW, Temkin NR, Dikmen SS, Winn HR: Side effects and mortality associated with use of phenytoin for early posttraumatic seizure prophylaxis. J Neurosurg 91:588-592, 1999

12. Holmes GL: Effects of seizures on brain development: lessons from the laboratory. Pediatr Neurol 33:1-11, 2005

13. Inaba K, Menaker J, Branco BC, Gooch J, Okoye OT, Herrold $\mathrm{J}$, et al: A prospective multicenter comparison of levetiracetam versus phenytoin for early posttraumatic seizure prophylaxis. J Trauma Acute Care Surg 74:766-773, 2013

14. Jones KE, Puccio AM, Harshman KJ, Falcione B, Benedict N, Jankowitz BT, et al: Levetiracetam versus phenytoin for seizure prophylaxis in severe traumatic brain injury. Neurosurg Focus 25(4):E3, 2008

15. Khan AA, Banerjee A: The role of prophylactic anticonvulsants in moderate to severe head injury. Int J Emerg Med 3:187-191, 2010

16. Kochanek PM, Carney N, Adelson PD, Ashwal S, Bell MJ, Bratton S, et al: Guidelines for the acute medical management of severe traumatic brain injury in infants, children, and adolescents-second edition. Pediatr Crit Care Med 13 (Suppl 1):S1-S82, 2012

17. Lewis RJ, Yee L, Inkelis SH, Gilmore D: Clinical predictors of post-traumatic seizures in children with head trauma. Ann Emerg Med 22:1114-1118, 1993

18. Liesemer K, Bratton SL, Zebrack CM, Brockmeyer D, Statler KD: Early post-traumatic seizures in moderate to severe pediatric traumatic brain injury: rates, risk factors, and clinical features. J Neurotrauma 28:755-762, 2011

19. Nau KM, Divertie GD, Valentino AK, Freeman WD: Safety and efficacy of levetiracetam for critically ill patients with seizures. Neurocrit Care 11:34-37, 2009
20. O’Neill BR, Handler MH, Tong S, Chapman KE: Incidence of seizures on continuous EEG monitoring following traumatic brain injury in children. J Neurosurg Pediatr 16:167176, 2015

21. Park JT, Chugani HT: Post-traumatic epilepsy in childrenexperience from a tertiary referral center. Pediatr Neurol 52:174-181, 2015

22. Rowe AS, Goodwin H, Brophy GM, Bushwitz J, Castle A, Deen D, et al: Seizure prophylaxis in neurocritical care: a review of evidence-based support. Pharmacotherapy 34:396-409, 2014

23. Taylor CA, Greenspan AI, Xu L, Kresnow MJ: Comparability of national estimates for traumatic brain injury-related medical encounters. J Head Trauma Rehabil 30:150-159, 2015

24. Tilford JM, Simpson PM, Yeh TS, Lensing S, Aitken ME, Green JW, et al: Variation in therapy and outcome for pediatric head trauma patients. Crit Care Med 29:1056-1061, 2001

25. Vavilala MS, Kernic MA, Wang J, Kannan N, Mink RB, Wainwright MS, et al: Acute care clinical indicators associated with discharge outcomes in children with severe traumatic brain injury. Crit Care Med 42:2258-2266, 2014

\section{Disclosures}

Dr. Wainwright reports a consultant relationship with Sage Therapeutics. Dr. Giza reports receiving grants/research support from NIH, NCAA, DoD, Today's and Tomorrow's Children Fund, UCLA Brain Injury Research Center, UCLA Faculty Grants Program, UCLA Steve Tisch BrainSPORT Program, and NFL-GE; serving as a consultant for the NFL-Neurological Care Program, NHLPA, Pearson PLC, Alcobra, and Neural Analytics Inc.; serving on advisory panels for LoveYourBrain, MLS, NBA, NCAA, and USSF; medicolegal relationships involving 1 or 2 cases annually; serving on the speakers bureau for the Medical Education Speakers Network; and having been commissioner of the California State Athletic Commission.

\section{Author Contributions}

Conception and design: Vavilala, Ostahowski, Kannan. Acquisition of data: Ostahowski. Analysis and interpretation of data: Vavilala, Ostahowski, Kannan, Qiu. Drafting the article: Ostahowski, Kannan. Critically revising the article: Ostahowski, Wainwright, Mink, Groner, Bell, Giza, Zatzick, Ellenbogen, Boyle, Mitchell. Statistical analysis: Qiu.

\section{Supplemental Information \\ Online-Only Content}

Supplemental material is available with the online version of the article.

Supplementary Figure. http://thejns.org/doi/suppl/10.3171/ 2016.4.PEDS1698.

\section{Correspondence}

Monica S. Vavilala, Department of Neurological Surgery, University of Washington, Harborview Injury Prevention and Research Center, 325 Ninth Ave., Box 359960, Seattle, WA 98104-2499. email:vavilala@uw.edu. 